\title{
Letter: The Idea of Using Botulinum Toxin to Treat Anxiety Disorders
}

\author{
Taleb Al Abdulmohsen
}

Department of Psychiatry, Ernst-Moritz-Arndt Uiversity, Lindenallee 24, 18437 Stralsund, Germany

Received 2012-08-04; Revised 2012-08-04; Accepted 2013-05-07

\begin{abstract}
After botulinum toxin was shown to help treat depression, I asked whether it can be therapeutic for anxiety disorders as well. In this brief letter, I go beyond pointing out my pioneering role in the suggesting of this idea and into highlighting some intuitive and counterintuitive aspects of this topic and some misconceptions. For example, if it is true that the effect of botulinum toxin occurs through the interruption of one's ability to express, and as a result to perceive, sad emotions, then why is it not that common to report a loss of sad feelings after having botulinum toxin injected as a cosmetic in exactly the same way as applied to treat depression?
\end{abstract}

Keywords: Botulinum Toxin, Anxiety Disorders, Treat Depression

\section{INTRODUCTION}

I was happy to read that Wollmer et al. (2012) had successfully replicated the results previously reported by Finzi and Wasserman (2006), showing that botulinum toxin can treat depression. However, it was unpleasant to discover that the authors had included my idea regarding the possible therapeutic value of botulinum toxin for anxiety disorders when at the time of publication I had been working for two years with both principle investigators, Wollmer et al. (2012) and Kruger et al. (1998), on the preparatory phase of a study conceptualized by me and funded by my country Saudi Arabia to test my own hypothesis that botulinum toxin could help treat social anxiety. Upon expressing my frustration to them, the initial response of Dr. Kruger was defensive, claiming that this was not a novel idea and citing irrelevant sources, while Wollmer et al. (2012) remained silent. It was not until I raised the subject with the committee of good clinical practice in Hannover Medical School that they both apologized and acknowledged in writing my priority of suggesting this idea.

However, they tried to rationalize their mistake by suggesting that my idea was published in their paper because it was simply a logical conclusion inferred from the context. This latest claim contradicts a number of facts that could be interesting to the reader. About one year before submitting their article, I informed Dr. Kruger about what could be the first published evidence of a relationship between the cosmetic use of botulinum toxin and psychiatric disorders. This was a 44-year-old woman who developed a severe anxiety reaction and what appears from the context to be a secondary depression after receiving botulinum toxin as a cosmetic treatment. The authors concluded that the loss of control of muscular activity of the forehead may have triggered an anxiety response similar to the one the patient had 24 years earlier (Brenner et al., 1999) and about two years before Wollmer et al. (2012) submitted their article, I informed Dr. Kruger about several self-reports I found online about patients who developed new, or a worsening of, anxiety symptoms after being treated with botulinum toxin. Moreover, both Wollmer et al. (2012) and Dr. Kruger are aware of anecdotal clinical evidence suggesting that the classical cosmetic procedure with botulinum toxin could cause a worsening, or at best a statistically non-significant improvement, of anxiety symptoms (this anecdotal evidence appeared in the framework of the above-mentioned study conceptualized by me). The only explanation I could think of for them to rush to suggest an idea contradicting the already available evidence and not belonging to them is that they 
based their hope on a slightly different procedure I suggested and they refused to apply, thinking that if this novel procedure proves to be successful in the future they would be touted as the first discoverers. You can add to this that when they submitted their paper they were fully aware of my interest in applying for a patent for a new medical use.

It is also interesting that no other researcher over the many years of addressing the use of botulinum toxin in the field of psychiatry has reached this "logical conclusion." On the other hand, many intuitive thoughts about this topic remain untouched. For example, Wollmer et al. (2012) echo a question of whether the attained therapeutic effect of botulinum toxin in alleviating depression is centrally due to retrograde transport or peripheral. However, they don't simply suggest differentiating this with an exclusively peripheral denervation method, like surgical (Yang et al., 2010), chemical (e.g., ethanol) (Yoshida, 2003), thermal (Kim et al., 2011), or cryic (Palmer et al., 2011) denervation, most of which are already in use to attain the same cosmetic effect achieved by botulinum toxin. Another intuitive question is, if it is true that botulinum toxin treats depression through the interruption of the muscular proprioceptive signals from muscles responsible for expressing sadness, then why do the millions of people who receive botulinum toxin in the glabellar area not lose their ability to feel sadness? Some people with apparently no history of depression do actually develop a partial loss of their ability to feel normal sadness after cosmetic botulinum toxin procedure, like the case self-reported by Ritvo (2010). But there is no report of total loss of sad feelings. This could be explained by saying that the muscular proprioceptive input forms only one component of emotion, responsible for some or all of the unwanted excessive sadness and some but not all of the normal sadness. Another explanation is that the absence of the proprioceptive limiting negative feedback (i.e., the one which tells the brain that there is enough muscular tension) could lead to an increase in the motor drive sent from brain to all muscles responsible for expressing sadness, including bodily and perioral muscles, which compensates for the lost glabellar proprioception and maybe also the lost component of emotion.

As far as wording is concerned, the original hypothesis, which indicates that the somatic sensory input could alter emotions, should I suggest be called the emotion modulating proprioception hypothesis (one can also call it, albeit less properly, the somatic feedback hypothesis). The use of the word "proprioception" instead of the word "feedback" is important to include sensory input in the absence of a centrally originating motor drive; for example, when stimulating muscles with external electricity as in this case, there would be no feedback but only a proprioception. The facial feedback hypothesis should also be called the facial primacy hypothesis, because it is concerned with emphasizing the primacy of facial muscles (Adelmann and Zajonc, 1989). Furthermore, Wollmer et al. (2012) suggest denervating some perioral muscles in patients with depression, but they stop short of saying that if treating perioral muscles fails to alleviate depression on its own, as achieved in the glabellar area, this will lead to a glabellar primacy hypothesis (glabellar feedback hypothesis), or a periorbital primacy hypothesis if the suborbital area is found to have an important role too.

Davis et al. (2010) differentiate the productive effect, which is "the mental processes engaged in producing an expression and/or in flexing facial muscles," from the reactive effect, which is "the feedback from the face once a facial movement occurs or an expression is formed." I would suggest reclassifying the effects into centrifugal/productive and centripetal/proprioceptive. The latter could further be classified into non-feedback, a proprioception in the absence of a centrally originating motor drive and feedback, reporting to the brain after receiving a motor signal from the brain. The non-feedback effect could further be classified into reactive (due to external stimulus) and non-reactive (due to a factor in the muscle itself). It must be emphasized here that the productive effect lies outside the general hypothesis that I called emotion modulating proprioception (somatic feedback hypothesis). The importance of this classification comes from another intuitive question, which is whether it could be that the etiology of a subtype of depression lies inside the facial muscles. Hypothetically, a local pathological factor in the muscle itself could be responsible for the overactivity or increased tension. In this case the non-reactive non-feedback effect would be the relevant one.

Using the proper term can ensure a better understanding. For example, Wollmer et al. (2012) consider James' statement "refuse to express a passion and it dies" a referral to the facial feedback hypothesis (facial primacy hypothesis) when it actually concerns the more general emotion modulating proprioception hypothesis. Moreover, the word "refuse" in James' statement implies the productive effect, although James apparently assumes that a successful prevention of expression will ultimately ensue and elicit (positively 
modulate) the proprioceptive effect which was his main interest. In fact, Grob (2009) has shown that employing the productive effect through voluntarily suppressing facial expressions has resulted in reboundly intensifying rather than ameliorating negative emotions.

With the introduction of topical botulinum toxin (Glogau et al., 2012), one should expect an expansion of the use of this method of denervation because of the ease of application. As botulinum toxin already found its way in the field of psychiatry, we should be ready for this new agent in the psychotropic family.

\section{CONCLUSION}

For many current writers, the delight of addressing the novel use of botulinum toxin as an emotion-altering substance is reduced in the affirmation of the longsuggested counterintuitive interplay between muscles and emotions, while balking at conceptualization and the introspective approach that the early writers enjoyed. Furthermore, modern instrumentalization has reduced the diagnostic process into a checklist or a logbook rather than a topology of symptoms. This could lead to the viewing of psychiatric disorders, at least at the practical level, as a collection of unrelated symptoms. This is a pattern in which the indulgence is unlikely to broaden thinking to see that this paper's topic is way beyond a simple relation between frown muscles and sadness. If we, for example, shift the subject to anxiety we should not underestimate the role of extraocular muscles responsible for moving eyes and creating the typical irritable gaze of anxious people, which is a point likely to be overlooked when tying oneself to the thinking of static expressions and prior to that, one should not forget the possible importance of the muscles of the vocal apparatus responsible for auditory expression as well as a possible direct muscular proprioception, both of which could be modulated using denervation. What could happen if we paralyze the muscle(s) responsible for vocally expressing sadness? Or if we dampen the muscle(s) responsible for the vocal flutter characteristic of anxiety in order to allow them to function without major oscillations?

Amid the celebration of the finding that botulinum toxin can treat depression, the word "proprioception" in this context has not yet been thoughtfully examined. The proprioceptive function is usually defined as the perception of position, which applies perfectly to joints. But in the case of muscles, we need to reemphasize the fact that proprioception is a sensation of position as well as stretch and tension and particularly in the facial region, proprioception is not only the awareness that a certain muscle is occupying, or has moved to, a certain position, but also a direct sensation of the state or alteration of a muscle's internal tension. Otherwise, any patient with depression would be able to alter his expressions and feel happy through external manipulation applied with fingers to the glabellar and perioral muscles. This fallacy has led once to the thinking that facial feedback hypothesis has been disconfirmed (Tourangeau and Ellsworth, 1979). In the external manipulation method there is an application of an external tension without any evidence or theoretical postulation indicating that the internal tension has been significantly altered. Voluntary smiles can be divided into spontaneous, non-spontaneous and resembled. The latter is an oral attitude without the intention to smile but which resembles a smile, like the case of holding a pen between one's teeth. Although the distribution and strength of the oral muscles' tension is not expected to be perfectly identical in the three cases of voluntary smile, in all of them it is obvious that smile agonists are shortened with internal tensions, which is absent in the case of externally manipulated smiles.

The overactivity of frown muscles that has been therapeutically targeted in patients with depression may theoretically be central or peripheral in origin. In both types, a subtype of this overactivity could be an over reactivity (hyperexcitability), in which case the muscle remains at rest if not stimulated but responds in an exaggerated manner to various stimuli. This could be the case in depressed patients who retain the capacity for appropriate mood fluctuation: their frown muscles are overreactive and so they overrespond to minor sad thoughts, expressing this minor sadness as if it were a major one, and then through muscular feedback to the brain the original minor sadness would be felt as an intensified one. This concept is more important when applied to anxiety disorders. What if a constellation of phobic muscles becomes overreactive in a child? Whether the first instigation comes from a horse or a rabbit, the overreactive phobic muscles of this child would exaggerate the otherwise normal childish fear beyond the adaptive behavioral learning process, creating the first symptoms of a lifelong specific phobia. A set of symptoms could then follow in a pursuit of finding a self-explanation and what if the over reactivity impacts the muscles responsible for expressing sexual appetite while the most attentive feminine figure facing the affected male child is his mother? Could this create an Oedipus complex? In other words, could the Oedipus complex and the psychodynamics built on it be, at least in part, a result of the muscular expressive exaggeration 
of an otherwise subthreshold thought that usually passes unnoticed or goes forgotten? And could the Oedipus complex be a person-specific disorder treatable with denervation?

The over reactivity I suggest could arise from exclusively biological factors, but could also be a learned pattern, or a combination of both. In this sense, psychological factors could "train" muscles to exaggerate certain expressions either generally or in particular conditions. This learned pattern is not the appearance but rather the intensity of the expression triggered by certain stimuli and this intensity is not the one proportional to a current or conditioned intense psychological stressor, but the one achieved through the repetition of a well-coordinated intense movement of a group of muscles.

Eight years ago I dreamed of carrying out a series of major studies testing, among other things, the use of botulinum toxin to treat psychiatric disorders. What happened next represents the pinnacle of failed cooperation. In October 2008, during my pursuit to carry out a study to test the effect of botulinum toxin on anxiety disorders or depression, I sent an email, with references mostly to depression but also to anxiety in its general sense, to Prof. Uwe Hartmann from Hannover Medical School. His answer showed no interest and gave no indication that the idea is already being considered by anyone in his department. The coincidence becomes obvious if we know that Prof. Hartmann is the direct boss of Dr. Kruger, who one year later initiated with Dr. Wollmer the first randomized controlled trial on botulinum toxin for depression and rejected, despite our clinical conjunction and parallel work on my own project, my repeated requests to contribute to the writing of their study article, in which my idea regarding anxiety was included without my permission or prior awareness. They also included the idea of treating perioral muscles in patients with depression. In the email that I sent to Prof. Hartmann I attached an unpublished article in which I suggested in clear words, in the context of treating depression with botulinum toxin, the inclusion of all overactive muscles beyond the ones treated in the study by Finzi and Wasserman (2006). I was referring not only to the perioral area, as they probably understood, but also the suborbital, lateral canthal and frontal areas.

For more details on my ideas regarding the effect of denervation on anxiety I refer the reader to my recently published paper (Al Abdulmohsen, 2013).

\section{REFERENCES}

Adelmann, P.K. and R.B. Zajonc, 1989. Facial efference and the experience of emotion. Ann. Rev. Psychol., 40: 249-80. DOI: 10.1146/annurev.ps.40.020189.001341

Al Abdulmohsen, T., 2013. The cosmetic use of intramuscular ethanol and its anxiety instigating and anxiety curbing side effects. UNAIS.

Brenner, R., S. Madhusoodanan, Z. Korn and M. Spitzer, 1999. Acute anxiety and depression induced by loss of sensation and muscle control after botulinum toxin a injection. Southern Med. J., 92: 738-738.

Davis, J.I., A. Senghas, F. Brandt and K.N. Ochsner, 2010. The effects of BOTOX injections on emotional experience. Emotion, 10: 433-440. DOI: $10.1037 / \mathrm{a} 0018690$

Finzi, E. and E. Wasserman, 2006. Treatment of depression with botulinum toxin A: A case series. Dermatologic Surgery, 32: 645-650. DOI: 10.1111/j.1524-4725.2006.32136.x

Glogau, R., A. Blitzer, F. Brandt, M. Kane and G.D. Monheit et al., 2012. Results of a randomized, double-blind, placebo-controlled study to evaluate the efficacy and safety of a botulinum toxin type a topical gel for the treatment of moderate-to-severe lateral canthal lines. J. Drugs Dermatol., 11: 38-45.

Grob, J.D.M., 2009. Dial E for Emotion: Context and consequences of emotion regulation. Dissertation. University of Groningen.

Kim, J.H., J.W. Jeong, D. Son, K. Han and S.Y. Lee et al., 2011. Percutaneous selective radiofrequency nerve ablation for glabellar frown lines. Aesthetic Surgery J., 31: 747-55. DOI: 10.1177/1090820X11416807

Kruger, T., M.S. Exton, C. Pawlak, A.V.Z. Mühlen and U. Hartmann et al., 1998. Neuroendocrine and cardiovascular response to sexual arousal and orgasm in men. Psychoneuroendocrinology, 23: 401-411. DOI: 10.1016/S0306-4530(98)00007-9

Palmer, F., V. Narurkar, T. Munyon and K. Tatsutani, 2011. Cold-induced modulation of targeted facial nerves. Proceedings of the 31st Annual Conference of the American Society for Laser Medicine and Surgery (ASLMS), Apr. 3.

Ritvo, E., 2010. Botox: A cure for depression? Psychology.

Tourangeau, R. and P.C. Ellsworth, 1979. The role of facial response in the experience of emotion. J. Pers. Soc. Psychol., 37: 1519-1531. PMID: 501520 
Wollmer, M.A., C. De Boer, N. Kalak, J. Beck and T. Götz et al., 2012. Facing depression with botulinum toxin: A randomized controlled trial. J. Psychiatric Res., 46: 574-581. DOI: 10.1016/j.jpsychires.2012.01.027

Yang, N.Z., B. Wang, Z.J. Wang, C. Zhang and X.K. Ma et al., 2010. Angular nerve of facial nerve: Anatomic research. Zhonghua Zheng Xing Wai Ke Za Zhi, 26: 221-225. PMID: 20737954
Yoshida, K., 2003. Muscle afferent block in the treatment of oromandibular dystonia. Difference in effect between masticatory and lingual muscles. Nervenarzt, 74: 516-522. PMID: 12799790 